\title{
Fluocinolone Implant for Idiopathic Retinal Vasculitis, Aneurysms, and Neuroretinitis Syndrome: A Case Report
}

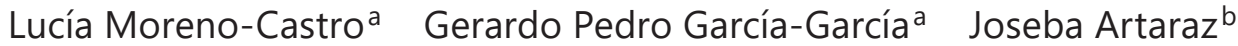 \\ Alex Fonollosa ${ }^{c}$ \\ aDepartment of Ophthalmology, Alicante University General Hospital, ISABIAL, Alicante, \\ Spain; ${ }^{b}$ Department of Ophthalmology, BioCruces Bizkaia Health Research Institute, \\ Cruces University Hospital, University of the Basque Country, Barakaldo, Spain; 'Bilbao \\ Ophthalmology Institute, Bilbao, Spain
}

\section{Keywords}

Idiopathic retinal vasculitis, aneurysms and neuroretinitis syndrome $\cdot$ Fluocinolone $\cdot$ ILUVIEN

\begin{abstract}
Idiopathic retinal vasculitis, aneurysms, and neuroretinitis (IRVAN) syndrome is a rare and progressive disorder that predominantly affects both the eyes of young female individuals and can threaten visual function. Peripheral ischemia and macular exudation are common findings in patients. The treatment options include panretinal photocoagulation (PRP), systemic immunosuppression, and intravitreal antiangiogenic and corticosteroid therapy. Fluocinolone acetonide intravitreal implant is approved for the treatment of nonanterior noninfectious uveitis and diabetic macular edema (ME), with an estimated therapeutic duration of 3 years. We describe a case of IRVAN syndrome in a child with ME who had been previously treated with PRP, antiangiogenic therapy, and several dexamethasone intravitreal implants and received a fluocinolone acetonide intravitreal implant in her right eye. The patient showed stabilization of the visual acuity and a marked reduction of the macular thickness 1 month after the treatment. At 12-month follow-up, the patient required perifoveal focal photocoagulation due to a rebound of the ME. After 2 years of follow-up, visual acuity remains stable and macular retinal thickening under control. Local long-standing steroid therapy has proved to be quite efficient in controlling the progression of the disease in our patient.
\end{abstract}




\section{Introduction}

Idiopathic retinal vasculitis, aneurysms, and neuroretinitis (IRVAN) syndrome is a rare condition, usually bilateral, which affects young patients, between 30 and 40 years, with a female preponderance [1-3]. It was first described by Chang et al. [3], and he proposed the acronym for its name. Samuel et al. [2] established a five-stage classification using major and minor criteria. Major criteria are retinal vasculitis, aneurysmal dilatations, and neuroretinitis; minor criteria are peripheral capillary nonperfusion, retinal neovascularization, and macular exudation.

Clinical presentation of this entity is variable; in fact, it can be asymptomatic or present with serious visual impairment [3]. Diagnosis of IRVAN is made in the absence of systemic pathology together with funduscopic typical abnormalities. The presence of cells in the anterior chamber or slight vitritis has been described [1,3]. Reported treatment options include panretinal photocoagulation (PRP) for the ischemic complications, systemic steroids and immunosuppression for the intraocular inflammation, and intravitreal injections of antiangiogenic agents and local steroids for the macular exudation and edema [1].

We describe a case of IRVAN syndrome in a child that presented macular edema (ME) managed with a fluocinolone acetonide intravitreal implant. Before this treatment, the patient had received periocular triamcinolone, intravitreal bevacizumab, and several dexamethasone intravitreal implants.

\section{Case Report}

A 12-year-old girl was referred to our medical center with a diagnosis of bilateral neuroretinitis and vasculitis. She had no history of previous systemic diseases. An ocular examination revealed a best-corrected visual acuity (BCVA) of 20/20 in the right eye (RE) and 20/40 in the left eye (LE). Intraocular pressure was $12 \mathrm{~mm} \mathrm{Hg}$ in both eyes; there was no inflammation in the anterior chamber, but mild vitritis, vasculitis, papillitis, and macular exudates in the fundoscopy exam were observed (shown in Fig. 1a-c). A fluorescein angiography showed peripheral ischemia and macroaneurysms (shown in Fig. 1d). Several tests had previously been performed, including a purified protein derivative test which resulted negative; infectious serologies including treponemic serology, Bartonela henselae serology, Toxoplasma gondii serology, and Borrelia burgdorferi serology, all of them negative; brain magnetic resonance imaging and chest X-ray, which were normal; HLA-B51 was positive and antinuclear antibodies at 1/50 titer. There was no history of oral or genital ulcers, joint inflammation, or cutaneous hypersensitivity that were suspicious for Behcet disease. No fever or pulmonary or cutaneous manifestation was present at presentation. Her pediatrician ruled out any systemic autoimmune disease.

Due to the typical clinical findings and because other etiologies were ruled out, a diagnosis of IRVAN syndrome was established. Treatment was initiated with oral corticosteroids of $30 \mathrm{mg}$ at tapering doses, and PRP was performed. Three months later, with the patient off steroids, worsening of BCVA (20/50 and 20/60 in RE and LE respectively) and ME was observed, and it was treated with 2 periocular triamcinolone injections and one intravitreal injection of bevacizumab without success. Hence, a dexamethasone implant was administered in both eyes with improvement in OCT (shown in Fig. 2a-d) and slight recovery in BCVA (20/40 and 20/50). The dexamethasone implant was effective, but recurrence of the ME occurred, so during the following 4 years, 9 dexamethasone implants were given in each eye. The patient developed a very mild posterior subcapsular cataract and ocular hypertension in both eyes after the first injection $(26 \mathrm{~mm} \mathrm{Hg}$ ), which was well-controlled with timolol drops.

\section{Karger'}




\section{Case Reports in Ophthalmology}
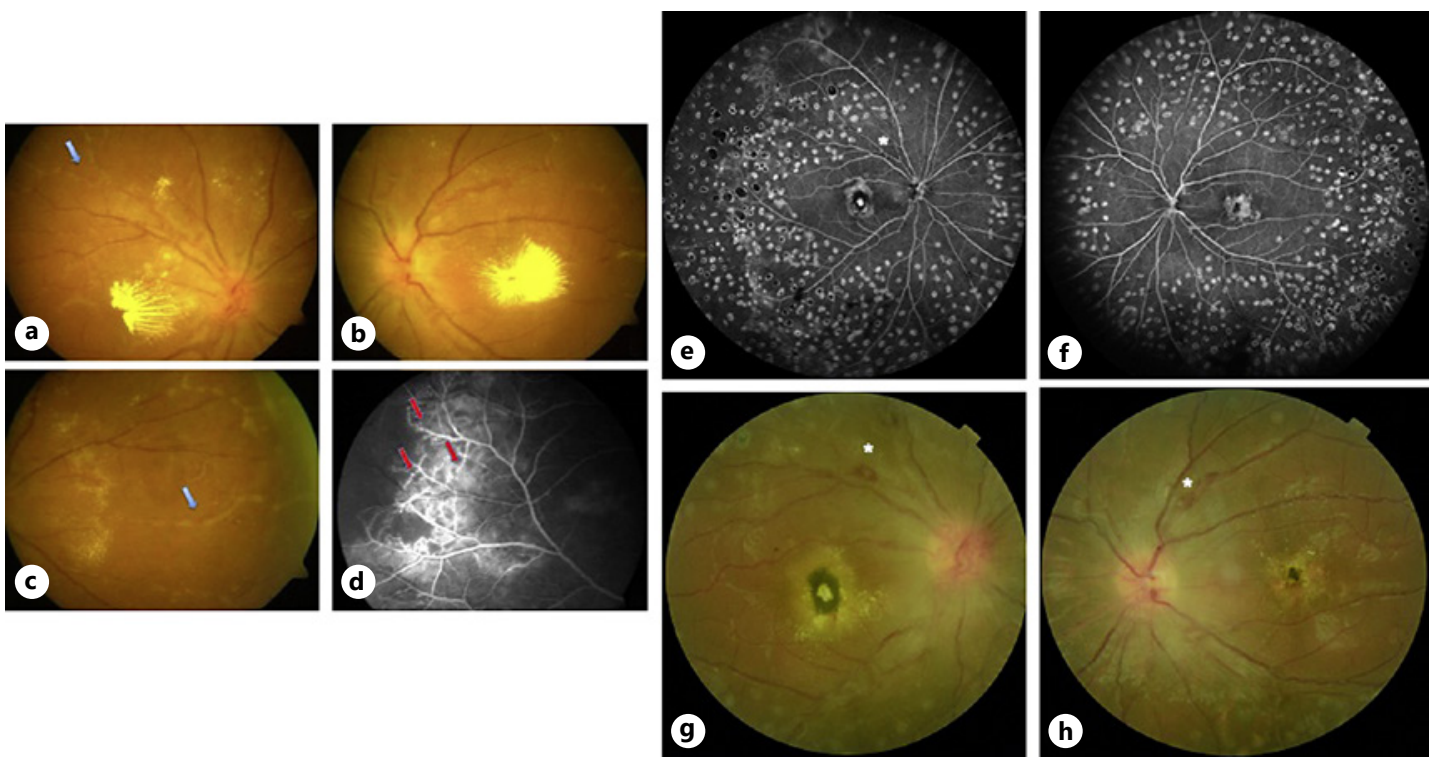

Fig. 1. a-c Retinographies at baseline; blue arrows show sheathing of arteries. $\mathbf{d}$ FAG; red arrows show macroaneurysms; an area of ischaemia is observed. e-h Imaging performed at 5-year follow-up visit. e, f FAG showing very mild leakage in the superior-temporal area of the RE; asterisk shows fusiform dilatations of arteries. g, h Retinographies where asterisks are pointing macroaneurysms with haemorrhages. FAG, fluorescein angiography; RE, right eye.

During follow-up, control retinographies and fluorescein angiography were performed in order to rule out development of neovascularization, which never happened (shown in Fig. 1e-h).

Because of the availability of fluocinolone acetonide intravitreal implant at our institution, we offered the patient this treatment in order to avoid frequent visits and injections. Previous authorization from the pharmacy department of our center was obtained. First, we injected it in her RE to assess effect. The appearance of the macula before the injection is shown in Figure 2e. A decrease in macular thickening was observed at 1 month which maintained at 6 months; at 12 months, a localized perifoveal increase was observed, which was successfully managed with a focal laser. At 2 years of follow-up, BCVA is 20/30 and 20/60 in the RE and LE, the retinal thickening remains stable (shown in Fig. 2g), and intraocular pressure is $16 \mathrm{~mm} \mathrm{Hg}$ in both eyes with topical treatment. Remarkably, a decrease in the macular retinal thickening of the LE was also observed after the fluocinolone acetonide intravitreal implant, which has remained until the last follow-up visit (shown in Fig. 2h). Though retinal thickening has remained stable, recovery of visual acuity has been limited by chronic intra- and subretinal lipidic exudates. Evolution of central macular thickness and VA during the 3 years before fluocinolone acetonide injection (while dexamethasone implants were given) and during the 2 years after this implant was given are shown in Figure 3.

\section{Discussion}

IRVAN syndrome is a rare clinical entity characterized by the presence of aneurysms, retinal vasculitis, and neuroretinitis. Three major pathological phenomena occur: inflammation (vitritis, vasculitis), ischemia, and ME.

Systemic corticosteroids have been used to treat inflammation [3]; however, their efficacy is controversial $[4,5]$. Immunosuppressive agents such as mycophenolate, azathioprine, or 


\section{Case Reports in Ophthalmology}
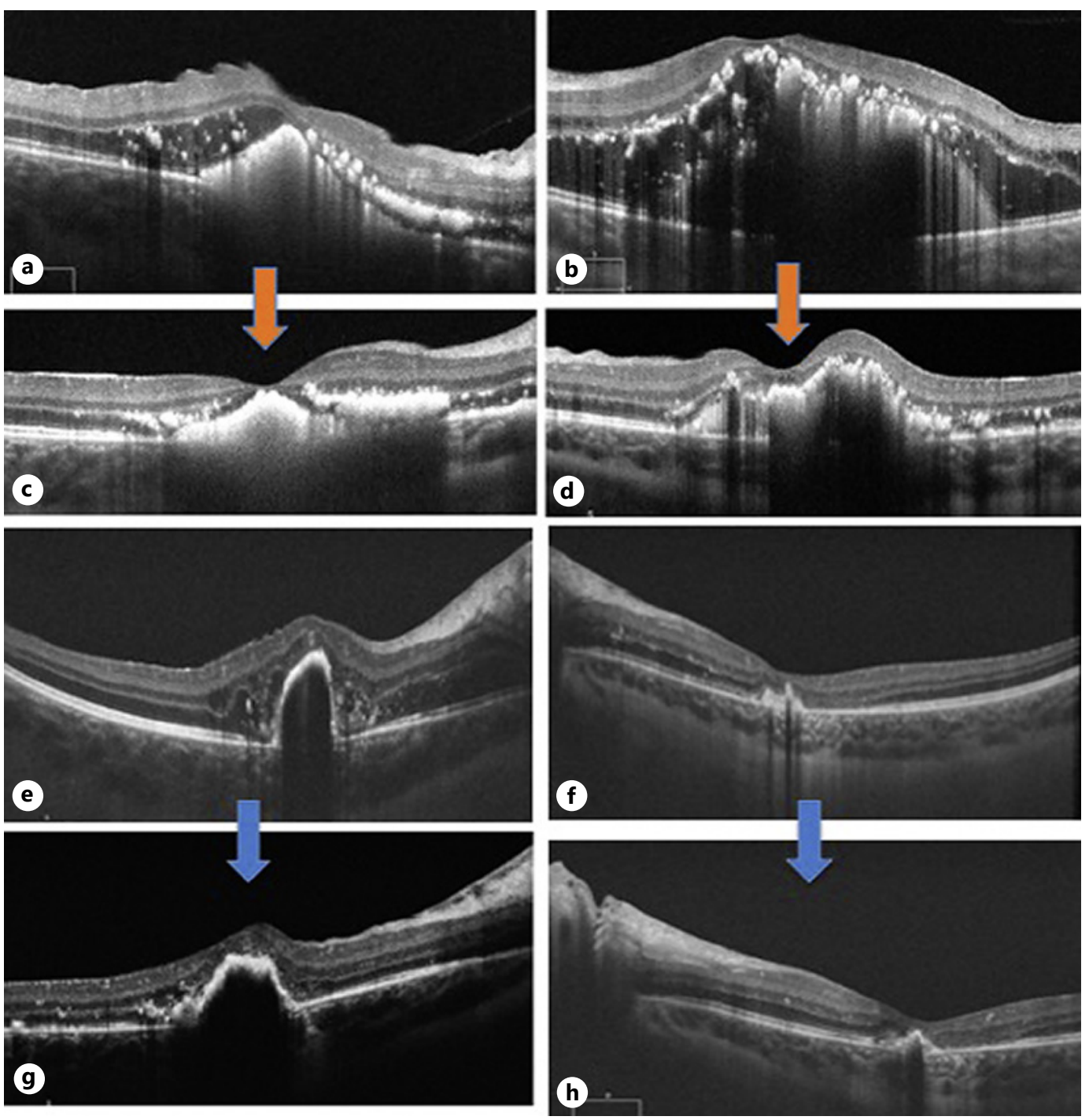

Fig. 2. OCT imaging. a-d Typical effect of dexamethasone implant. e, $\mathbf{f}$ OCT scans before fluocinolone implant. $\mathbf{g}, \mathbf{h}$ Scans after treatment (2 years of follow-up).

infliximab have been used to control retinal vasculitis and inflammation with satisfactory outcomes in reducing inflammation and ME [5-7], although Ameratunga et al. [5] suggest that immunosuppression might be a good option for early stages of IRVAN disease but less effective in advanced stages. In the present case, systemic corticoids were used for 3 months, achieving control of the inflammatory phenomena. Both ischemia and ME may be associated with poor visual outcomes in patients with IRVAN syndrome. In order to avoid the complications associated with the former, PRP is mandatory. Samuel et al. [2] classified IRVAN syndrome in 5 stages based on retinal ischemia: stage 1 , vasculitis, retinitis and macroaneurysms; stage 2 , nonperfusion in fluorescein angiography; stage 3 , posterior pole neovascularization, including vitreous hemorrhage; stage 4, anterior pole neovascularization; and stage 5, neovascular glaucoma. According to this classification, our patient was in stage 2 and, as recommended by Samuel et al. [2], PRP was performed. During 7 years of follow-up, no neovascular complications have developed.

Regarding the management of ME in IRVAN syndrome, several options have been reported: 


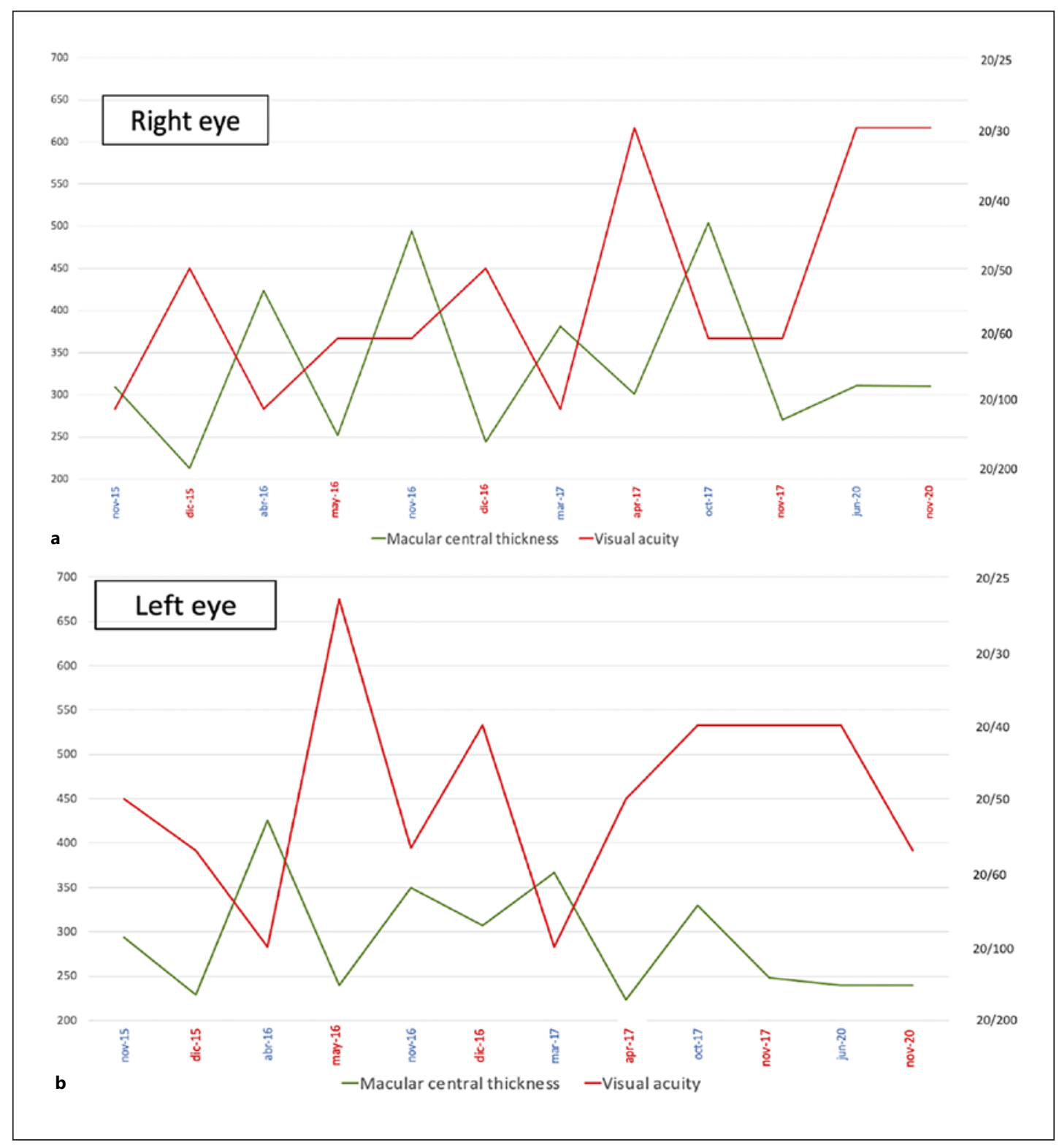

Fig. 3. Evolution of CMT and VA previous to fluocinolone injection ( 3 years) and after it (2 years). a RE. b LE; dates in blue color are the injection dates, and dates in red color are the control visit after the injection. RE, right eye; LE, left eye; CMT, central macular thickness.

Sawhney et al. [8] reported the use of intravitreal bevacizumab and dexamethasone implant in a patient with ME. The patient needed periocular triamcinolone injections and vitreoretinal surgery to bring the ocular inflammation under control.

Saatci et al. [6] reported resolution of ME and posterior pole exudates with a dexamethasone implant in a patient with systemic immunosuppressive treatment.

Empeslidis et al. [9] reported a case of persistent ME treated with a dexamethasone implant.

In IRVAN syndrome, macular thickening may be associated with both capillary hyperpermeability and vasculitis, and therefore, steroids, which are able to treat both phenomena, seem a very suitable option for this condition. Moreover, a local approach is possible because IRVAN is not associated with systemic disease. 
Fluocinolone acetonide intravitreal implant is an intravitreal implant of $0.19 \mathrm{mg}$ fluocinolone acetonide that releases $0.2 \mu \mathrm{g}$ per day during a period of 36 months [10]. This implant is approved for the treatment of diabetic ME and nonanterior noninfectious uveitis with an expected duration effect of 3 years.

In our case, since we had observed that intravitreal steroids had been effective in managing ME though the effect was transient, we thought that a fluocinolone intravitreal implant might be useful. Except for a localized recurrence that was successfully treated with focal laser, macular thickening has remained stable for 2 years. Unfortunately, recovery of visual acuity was limited due to chronic retinal lipidic exudates. A matter to note is the effect in the fellow eye. Though the mechanism of this effect is poorly understood, it has been described with other intravitreal treatments, dexamethasone implant [11], or antiangiogenic agents [12].

To our knowledge, this is the first case of IRVAN syndrome treated with a fluocinolone acetonide intravitreal implant. Because the condition is chronic, it has no associated systemic disease and both retinal inflammatory and hyperpermeability phenomena occur; we believe that an approach based on a local long-standing steroid is very reasonable in this disease.

\section{Statement of Ethics}

Ethics approval was not required for this case. The legal guardians of the patient have given their written informed consent to publish this case and any accompanying images. This study protocol was reviewed, and the need for approval was waived by Comité Ético de Investigación Clínica Hospital Universitario Cruces.

\section{Conflict of Interest Statement}

The authors have no conflicts of interest to declare.

\section{Funding Sources}

This manuscript received no funding support.

\section{Author Contributions}

Lucía Moreno Castro, principal author, wrote the manuscript. Gerardo P. García evaluated, improved, and corrected the manuscript. Joseba Artaraz, the patient's reference doctor, helped and supervised the project. Alex Fonollosa directed and supervised the development of the manuscript.

\section{Data Availability Statement}

All data generated or analyzed during this study are available in the text and its supplementary material files. Further inquiries can be directed to the corresponding author.

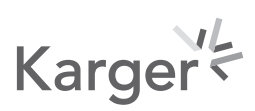




\section{References}

1 Bajgai P, Katoch D, Dogra MR, Singh R. Idiopathic retinal vasculitis, aneurysms, and neuroretinitis (IRVAN) syndrome: clinical perspectives. Clin Ophthalmol. 2017 0ct;11(9):1805-17.

2 Samuel MA, Equi RA, Chang TS, Mieler W, Jampol LM, Hay D, et al. Idiopathic Retinitis, Vasculitis, Aneurysms, and Neuroretinitis (IRVAN). New observations and a proposed staging system. Ophthalmology. 2007;114(8): 1526-30.

3 Chang TS, Aylward GW, Davis JL, Mieler WF, Oliver GL, Maberley AL, et al. Idiopathic retinal vasculitis, aneurysms, and neuro-retinitis. Retinal vasculitis study. Ophthalmology. 1995;102(7):1089-97.

4 Vichare N, Bhargava N, Brijmohan, Chaturvedi PK. A rare case of idiopathic retinal vasculitis, aneurysms and neuroretinitis (IRVAN) syndrome. Med J Armed Forces India. 2015;71:S257-60.

5 Ameratunga R, Donaldson M. Treatment of Idiopathic Retinal Vasculitis, Aneurysms, and Neuroretinitis (IRVAN) with photocoagulation in combination with systemic immunosuppression. Retin Cases Brief Rep. 2020 Dec 19;14(4):334-8.

6 Saatci AO, Ayhan Z, Takeş Ö, Yaman A, Bajin FM. Single bilateral dexamethasone implant in addition to panretinal photocoagulation and oral azathioprine treatment in IRVAN syndrome. Case Rep Ophthalmol. 2015;6(1):56-62.

7 Cheema RA, Al-Askar E, Cheema HR. Infliximab therapy for idiopathic retinal vasculitis, aneurysm, and neuroretinitis syndrome. J Ocul Pharmacol Ther. 2011;27(4):407-10.

8 Sawhney GK, Payne JF, Ray R, Mehta S, Bergstrom CS, Yeh S. Combination anti-VEGF and corticosteroid therapy for idiopathic retinal vasculitis, aneurysms, and neuroretinitis syndrome. Ophthalmic Surg Lasers Imaging Retina. 2013;44(6):599-602.

9 Empeslidis T, Konidaris V, Brent A, Vardarinos A, Deane J. Kyrieleis plaques in herpes zoster virus-associated acute retinal necrosis: a case report. Eye. 2013;27(9):1110-2.

10 Campochiaro PA, Nguyen QD, Hafiz G, Bloom S, Brown DM, Busquets M, et al. Aqueous levels of fluocinolone acetonide after administration of fluocinolone acetonide inserts or fluocinolone acetonide implants. Ophthalmology. 2013;120(3):583-7.

11 Pareja-Ríos A, Bonaque-González S. Unilateral intravitreal dexamethasone implant for diabetic macular edema: effect in the contralateral eye. Arq Bras Oftalmol. 2020;83(1):73-5.

12 Hanhart J, Tiosano L, Averbukh E, Banin E, Hemo I, Chowers I. Fellow eye effect of unilateral intravitreal bevacizumab injection in eyes with diabetic macular edema. Eye. 2014 May;28(6):646-53. 